\title{
Caracterización de la cultura afro como estrategia de educación ${ }^{1}$ Characterization of afro culture
as an education strategy
}

DOI: http://dx.doi.org/10.17981/cultedusoc.9.2.2018.10

Fecha de recepción: 09/06/2018. Fecha de aceptación: 04/09/2018

\author{
Aroldo Muñoz ${ }^{2}$ (iD \\ Edis Granados; Leila Villamil; \\ Lina Mayorga; Darwin Carbono y Pedro Pico \\ IED San Juan de Palos Prietos (Colombia) \\ maroldomuñoz@hotmail.com
}

Para citar este artículo

Muñoz, A., Granados, E., Villamil, L., Mayorga, L., Carbono, D. y Pico, P. (2018). Caracterización de la cultura afro como estrategia de educación. Cultura. Educación y Sociedad 9(2), 106-116. DOI: http://dx.doi.org/10.17981/cultedusoc.9.2.2018.10

\section{Resumen}

La presente investigación tuvo como objetivo demostrar la caracterización de la cultura afro como estrategia de educación. El desarrollo del estudio se enmarco en autores como Sánchez, Arrocha, bajo un corte descriptivo en tanto lo que se realiza es describir realidades. Esto es, decir cómo es y se manifiesta determinado fenómeno, de corte cualitativo. Los instrumentos aplicados fueron la observación y la entrevista estructurada a (40) estudiantes de diferentes cursos. Como resultado se pudo observar que la comunidad educativa de la IED San Juan de Palos Prieto resalta la importancia de celebrar el día de la Afro-Colombianeidad dado que somos una raza mestiza. Que el carácter Afro de la población mundial no es una cuestión exclusivamente de éstos, sino que es un patrimonio de la humanidad por lo que significa para el conocimiento universal.

Palabras clave: Caracterización, cultura, estrategia, afro, educación.

\begin{abstract}
The objective of this research was to demonstrate the characterization of Afro culture as an education strategy. The development of the study is framed in authors such as Sánchez, Arrocha, under a descriptive cut as what is done is to describe realities. "That is, saying what a certain phenomenon is like and what it is. Descriptive studies seek to specify the important properties of people, groups, communities or any other phenomenon that is subject to analysis. Measure or evaluate various aspects, dimensions or components of the phenomenon or phenomena to investigate to (40) students of different courses. As a result, it could be observed that the educational community of the IED San Juan de Palos Prieto highlights the importance of celebrating the day of Afro-Colombianeidad since we are a mestizo race. That the Afro character of the world population is not a matter exclusively of these, but that it is a patrimony of humanity for what it means for universal knowledge.
\end{abstract}

Keywords: Characterization, culture, strategy, afro, education.

\footnotetext{
${ }^{1}$ Este artículo ha sido derivado del Programa de Fortalecimiento de la Cultura Ciudadana y Democrática CT+I a través de la IEP apoyada en TIC en el Departamento de Magdalena: CICLÓN. Desarrollado con docentes miembros del Grupo de investigación "Palos prietos" pertenecientes a la Institución Educativa Departamental San Juan de Palos Prietos.

${ }^{2}$ Líder de investigación del grupo "Palos prietos".
} 


\section{Introducción}

La historia de los pueblos aborígenes a lo largo del tiempo ha sido la historia de la exclusión y la marginalidad, en ella los principales afectados sin duda han sido los indígenas y los pueblos afro. Ahora para nadie es un secreto que hoy existe conocimiento pleno de esa historia de marginalidad de exclusión social, sin embargo, muy a pesar de ello aún se presentan sistemáticamente practica que ignoran las conquistas históricas de estos gropos sociales. Sumado a esto, si bien el reconocimiento de derechos existe, las instituciones sociales de la sociedad moderna se han creado bajo un esquema fundamentalmente occidental, propias del hombre europea, para los europeos, es decir, nuestra historia institucional es la historia del espíritu europeo, lo cual, en esencia se constituye en una barrera frente a la cosmovisión del hombre afro.

La educación sin dada es expresión de ello, no obstante, solo en ella y a través de ella es posible repensar la genética cultural del hombre afro y extraer de él lo más valioso, lo más grande, que muy a pesar de su excluyente historia, tiene grandes valores por rescatar para construir una sociedad emancipada, incluyente, tolerante y sobre todo democrática. Creemos que en el colegio IED San Juan de Palos Prieto, tiene un sustrato de mucho valor de la cultura afro, que se haya en el olvido, es decir, que se sabe que está pero que se desconoce comprensivamente las aportaciones para la educación se podrían hacer, a partir de la riqueza de su cultura.

En otras palabras, no existe una caracterización plena de la riqueza cultural afro, su desconocimiento en consecuencia provoca un divorcio entre los modelos pedagógicos dominantes y la cultura de la minoría de sus habitantes, y con hablamos de estos, en efecto nos referimos a los niños y niñas que hacen parte de la comunidad educativa de Palos Prieto. Lo grave en todo esto es que los niños y niñas desconocen el origen de la cultura afro y se apropian de modas y patrones que poco aportan a su crecimiento integral, dejando de lado las raíces culturales y folclóricas autóctonas, perdiendo con ello valores, de tal forma que se ve amenazada sus tradiciones, sino la génesis de su cultura, generando con ello una profunda crisis de identidad.

En este sentido, el saber cultural del pueblo afro, a través de sus manifestaciones culturales, posibilitaría que dicha educación se ajustara al equilibro de una educación en contexto que responda a las necesidades de sus niños y niñas y no, una educación de estos a las pretensiones burocráticas de aquellos que en mejores condiciones viven recetando fórmulas de educación en escenarios descontextualizados. Por tal razón la presente investigación busca describir los elementos que caracterizan la cultura afrocolombiana que se constituyen en una estrategia de educación en la IED San Juan de Palos Prieto.

Uno de los principales grupos étnicos en Colombia víctima del conflicto armado interno son los afrodescendientes. Por ser víctimas actualmente y a través de la historia, tenemos suficiente potestad tanto política, ética y moral para decir que la violencia no es el camino correcto. Históricamente los daños sufridos por la esclavización y la sumisión de la violencia producto del conflicto armado nos han sido remediados; discutir sobre las victimas tanto afrocolombianas como de otra índole, debe hacerse desde el pasado hasta la 
actualidad. Los sufrimientos actuales de nuestro pueblo, están íntimamente ligados con los sufrimientos pasados, la expresión de la esclavización, es hoy el racismo y la discriminación racial, es la marginalidad y la exclusión a la cual hemos sido sometidos, no hay nada más violento que esta situación.

Los bailes, los peinados son propios de la comunidad afro, sus prácticas culturales son muy importantes y marcan un conjunto de tradiciones y creencias que se han movido a lo largo de la historia. En 1852 ganaron el derecho a ser libres tras la abolición de la esclavitud en nuestro país. Contribuyeron extraordinariamente desde el inicio en la cultura de Colombia. Los turbantes, los rasgos físicos, los peinados y la ideología son propios de esta comunidad. Al emplear la palabra Afro puede se puede remitir a una infinidad de pensamientos y caracteres, durante el desarrollo de este proyecto utilizamos el concepto refiriéndonos a las personas y culturas nativas del continente africano que hacen parte de la gran diversidad de complejos culturales como los Malinké, Ashanti, Bantú, Yoruba, Akan, entre otros, así como a sus dispersiones (Sánchez, 2017).

Genéricamente el uso de la categoría es impreciso al momento de evidenciar las diferencias que existen entre experiencias dancísticas, personas y comunidades. No obstante, el concepto fue utilizado puesto que la investigación tiene como propósito identificar la forma como se comunican y la forma como "danzas y culturas afro" está cargado de sentido por quienes las ponen en práctica (Sánchez, 2017).

Para Carvalho (2002, citado por (Sánchez, 2017), "se ha acrecentado la visibilidad y apropiación de la cultura afro en los centros urbanos occidentales.
Han emergido diferentes prácticas de danza, música y religión, ofrecidas como experiencias para los jóvenes". Asimismo, Arocha (2005, citado por (Sánchez, 2017), muestra que existe un "etnoboom de los patrimonios indígenas y afrocolombianos en el que, paradójicamente, existen procesos de despojo y erosión del patrimonio étnicoterritorial de estas comunidades, se promulga el reconocimiento de la diversidad, convirtiéndose en objetos exóticos para la canibalización globalizada" (Arocha, 2005, citado por (Sánchez, 2017).

La identificación de las personas y culturas afro y su representación, en estos procesos de globalización de símbolos e identidades, pareciera quedar simplificada y no responde al entendimiento de los contextos de donde provienen. En este sentido surgen las siguientes preguntas: ¿Cómo se presenta la apropiación de las prácticas culturales por quienes ejecutan danzas tradicionales del occidente de África? ¿Qué fundamenta el consumo cultural de dicha manifestación y a qué representaciones se asocia? (Sánchez, 2017).

En el continente americano la cultura afroamericana es una realidad confusa. Para ser identificada hay que hacer una reflexión sociológica y antropológica en el que interviene una visión de proceso, involucrando tiempo y espacio. El intelectual afrovenezolano Jesús "Chucho" García, (citado por (Antón, Bello, Del Popolo, Paixao, \& Rangel, Población y desarrollo, 2009). Define la afrodescendencia como el "resultado de un largo proceso de conservación-recreación y transformación de acuerdo a las condiciones socio-históricas y económicas que les ha correspondido vivir" (García, 2001).

De esta forma la cultura afrodescendiente se enmarca en fenómenos histó- 
ricos que perduran a través del tiempo, que se caracterizan por momentos de ruptura, continuidades, deconstrucción y reconstrucción (Antón, 2006). Se trata entonces de un paralelismo que es concebido como una contestación transcendental a la esclavización y a la necesidad de adaptación a nuevos contextos, como el de las Américas (Antón, Bello, Del Popolo, Paixao, \& Rangel, Población y desarrollo, 2009), Siguiendo a García, dentro del proceso de larga duración que significó la conformación de la cultura afrodescendiente en las Américas hubo dos características esenciales, la resistencia y la autoconciencia: "La africanía resistió dignamente en un acto de cimarronaje silencioso, buscando mecanismos de sobrevivencia" (García, 2001, citado por (Antón, Bello, Del Popolo, Paixao, \& Rangel, Población y desarrollo, 2009).

Dicho fenómeno intimó a romper con la conciencia ingenua y colonizada, asumiendo una conciencia crítica y descolonizadora, la cual fue el inicio para la revalorización cultural, la búsqueda de la libertad, la conquista de la ciudadanía, la politización de la identidad y luego la lucha frontal contra el racismo y la pobreza. Igualmente, al compartir inicios, cultura e identidad, los afrodescendientes enfrentan problemáticas sociales cuyos caracteres centrales han sido la esclavización, la colonización, la discriminación y la exclusión. Al menos así lo admitió la III Conferencia Mundial contra el Racismo, la Discriminación Racial, la Xenofobia y las Formas Conexas de Intolerancia, celebrada por las Naciones Unidas en Durban (Sudáfrica) durante 2001:

Se Reconoce que los afrodescendientes han sido durante siglos víctimas del racismo, la discriminación racial, y la es- clavización, y de la denegación histórica de muchos de sus derechos. Como consecuencia del racismo y la discriminación, estas comunidades han sufrido una serie de privaciones en la realización de sus derechos humanos, en especial los derechos económicos, sociales y culturales. P.P.

Por lo que la pobreza, la desigualdad socioeconómica y la marginalidad en gran parte de las sociedades afrodescendientes de América Latina se conviertan en una condición deshonrosa compartida.

Muchos son los autores que concuerdan en que el cuadro de desigualdad y limitaciones con los que se ven enfrentados los afrodescendientes se originó desde la colonia (Chalá, 2006); (Torre, 1996); (Rahier, 1998) citados por (Antón, Bello, Del Popolo, Paixao, \& Rangel, Población y desarrollo, 2009). Al inicio de la república como en la colonia, las sociedades latinoamericanas crearon un sistema jerárquico establecidos sobre pirámides raciales, donde las personas blancas y los mestizos se ubicaban en la cumbre, a una escala superior, gozando de privilegios como ciudadanos, por otro lado, en la parte inferior de la pirámide se encontraba los indígenas y los esclavos provenientes de áfrica, al igual que sus descendientes. La pirámide social se mantuvo por muchas épocas, incluyendo los tiempos del liberalismo radical.

En el periodo de la modernidad latinoamericana, afrodescendientes e indígenas observaron cómo fueron frustrados sus intenciones de ser libres, iguales y ciudadanos plenos. Los proyectos identitarios que giraban alrededor del mestizaje y la democracia racial sirvieron de base para la construcción de una identidad nacional, asimismo escondieron las discrepancias raciales, profundizando en el racismo y la discriminación. Esta rea- 
lidad, propia tanto de afrodescendientes y de indígenas, compone el argumento del racismo estructural que llevan sobre sus hombros e imposibilita que surjan de su situación de pobreza, desigualdad tanto personal como socioeconómica y exclusión social y política (Oliveira, 2001); (Stavenhagen, 2001), citados por Antón, Bello, Del Popolo, Paixão, \& Rangel, 2009).

En los tiempos de la colonización, el continente africano no fue visto como objeto de conocimiento por parte de los medios colombianos donde se producían los conocimientos. Durante este tiempo no se supo mucho de África por su lejanía, lo poco que se supo fue gracias a los viajeros, ensayistas y periodistas.

No obstante, la mayoría de los escritos forman un asiento de conocimientos cuestionados, por lo que ayudaron al fortalecimiento de los imaginarios peculiares sobre los esclavos que eran traídos de África Subsahariana e influyeron sobre el estilo y la calidad de las deliberaciones políticas seguidas durante la colonización española. A partir de la derogación oficial de la esclavitud hasta la mitad del siglo XX, los académicos desconocían la eficacia científica y política de estudios sobre los afrocolombianos, al igual que la del continente de donde (África) fueron traídos obligatoriamente. Incluso en los años treinta (siglo XX), la noción sobre África se siguió delimitando a descripciones vagas y poco profundas sobre los exploradores, los agentes coloniales, de parte de misioneros, etnólogos, etc.; descripciones elaboradas y narradas desde una perspectiva euro-centrista (espacio europeo). (Wabgou, 2008).

"La educación de los niños/as se realiza dentro de un contexto tradicional muy marcado por la enseñanza de la importancia del sentido y ejercicio de la solidaridad horizontal entre los miembros de la familia extensa" (Bartomeu, 1997). Traídos a la fuerza al continente americano, los esclavos transportaron consigo sus valores culturales (bagaje cultural) y rituales en su alma ya que estaban desposeídos de todos sus bienes materiales. Es por ello, que en su destino consiguieron hacer cara a la imposición total del catolicismo. Estos esclavos traían consigo recursos, legados por la esclavización en América Latina, acogen formas de clasificaciones políticas parecidas a las de sus ascendientes africanos. Es por eso su carácter guerrero y reivindicativo se acerca al de los africanos.

A través de la historia africanos y afrodescendientes fueron desterrados de sus tierras, como primera medida fue el desgarramiento de África y actualmente es de los lugares que los socorrieron al conquistar su libertad. los afrocolombianos poseen una condición de resistencia. Tenacidad frente a la expulsión de sus tierras y su negación como una situación natural impuesta. La separación territorial fue y procura seguir siendo impuesto por las élites imperiosos. La forma en la actualidad es doble, la primera es el embargo de los zonas que pertenecen a estas comunidades, especialmente los que habitan la costa pacífica, y el segundo es el destierro del conflicto armado en Colombia, que repercutió al igual que en la mayoría del territorio nacional, en las tierras (Andrade, 2011) donde vivían los afrocolombianos. A mediados de los años noventa las incursiones armadas se intensificaron, generando desplazamientos masivos a los centros urbanísticos del país.

El respeto por el desarrollo socioeconómico de las comunidades Afrocolombianas, conforme a su cosmovisión, hace referencia a las proyecciones de construc- 
ción de propósitos de vida comunitarios con sus propios recursos humanos y potencialidades culturales. Para el desarrollo social en estos aspectos también se denomina Etnodesarrollo. Asi mmisno, en el Plan Nacional de Desarrollo de la Población Afrocolombiana "Hacia una nación pluriétnica y multicultural”, se reconoce a las Comunidades Afrocolombianas su experiencia histórica y capacidad de autodeterminación para impulsar su desarrollo. (Ministerio de Educación Nacional. 2002, p. 20) (Mineducación, 2002).

"La población afrocolombiana y la lógica de su concepción de vida le permite vivir en armonía con la naturaleza y el otro, por lo tanto, es uno de los elementos que exige mayor y mejor atención del gobierno colombiano en contraprestación a la conservación de estos recursos". (Mineducación, 2002)

Ahora bien, El término "afro", que hace referencia a la ascendencia de una parte de la población, en Colombia y en otros países, evoca las raíces africanas (Palacios , Hurtado, \& Benítez, 2010). Una palabra que es utilizada hace poco tiempo. Su pronunciamiento crea controversia, ya que alude a modo de ver-se, en la pertenencia a un grupo social. Ahora bien, los términos afrocolombianos, afrocolombiana, afrocolombianidad se remiten a la historia geopolítica de las habitantes de las zonas del litoral Pacífico, primordialmente, y también a las que ocupan, en procesos de destierro e hibridación, las principales ciudades del país. A la expresión, "se la identifica con la ascendencia de las personas que fueron acarreadas en la trata esclava" (Ianini, 1975). Durante períodos a la expresión se caracteriza e identifica, sobre todo, irrespetuosamente, por la característica de ser de piel oscura, por sus rasgos fenotípicos, como "negro", "negra".

En la actualidad, alude al "hilo de unión" histórica con un continente apartado se incita a rescatar una identidad étnica en particular, una que no ha sido valorada, como lo exige la coyuntura social y política tanto en Colombia como el mundo. El desarrollo de actividades en el transcurso de sus vidas las personas afrodescendientes, hoy, se empiezan a conocer, por lo que se plantea, desde los movimientos sociales, la realización de indagaciones y ejercicios académicos, que conlleven a acciones y políticas públicas afirmativas, propensos a evolucionar hacia una actitud de interculturalidad, donde se hable de saberes y de experiencias multiétnicas, que aprendan a reconocer el valor de la persona $y$, en términos de igualdad y tolerancia, la importancia que tienen las expresiones de cada cultura, de quienes las conforman, en la construcción social y la diversidad que se manifiesta.

Varios autores han trabajado en este campo, uno de los que mejor ha definido el panorama y los antecedentes de las comunidades étnicas afrocolombianas o "negras" en nuestro país desde los años 1980, es el investigador Juan de Dios Mosquera (1986) citados por (Palacios , Hurtado, \& Benítez, 2010). Mosquera desde sus estudios de campo, ha relatado las situaciones de la intervención política y la entrada a los escenarios de gobierno, de una $\mathrm{u}$ otra forma, negado al pueblo descendiente de africanos en nuestro país. También abre la puerta a lo que es incuestionable hoy en día: escasas personas que pertenecen a comunidades étnicas afrocolombianas, mestizos e indígenas, han ascendido a altos oficios jerárquicos en la administración institucional. 
Incluso con casos que son excepcionales, es común ver como se relega a la población afro en Colombia, por lo cual llegan a ocupar solo cargos secundarios, sin tener la posibilidad para ser partícipes en las transformaciones necesarias de su medio social, y por la falta de políticas etno-educativas, unos pocos que pudieron un cargo de poder político, han optado por seguir los esquemas individualistas, convenientes en una sociedad capitalistas, racistas y machistas, con acciones partidistas y particulares, y no de servicio público (Palacios, Hurtado, \& Benítez, 2010).

\section{Metodología}

Esta investigación es de carácter descriptivo, por lo que permite identificar "características del objeto de estudio, señala formas de conducta y actitudes del universo investigado, establece comportamientos concretos y descubre $\mathrm{y}$ comprueba la asociación entre categorías de investigación”. (Miguelez, 2006). Es de enfoque cualitativo, debido a que lo que se pretende analizar es un hecho social que afecta de manera definible pero no cuantificable a la población Afrocolombiana.

El paradigma que mejor se adapta con la investigación es de índole cualitativo y de acuerdo a los objetivos que se persiguen, es el prototipo socio-crítico que apuntan a la transformación de las relaciones sociales y a brindar refutaciones las problemáticas procedentes de éstos.

\section{Escenarios y actores}

Los participantes fueron los estudiantes de la IED San Juan de Palos Prieto. En este sentido se escogieron cuarenta (40) en representación para el estudio.
Técnica e instrumentos de recolección de información

Se realizó observación y entrevistas a un grupo representativo de niños $\mathrm{y}$ niñas de la institución. El factor de inclusión que se tuvo en cuenta fue esencialmente el hecho de que se predica que en ellos se encuentra la principal amenaza de pérdida de identidad cultural afro.

\section{Procedimiento}

Momento (1): Elección del fenómeno a estudiar.

Momento (2): Hacer el sondeo diagnóstico de los objetos de interés.

Momento (3): Definición de las categorías de estudio: Cultura afro, Estrategia educativa.

Momento (4): Definición de los instrumentos de recolección de la información. Momento (5): Aplicación de la entrevista estructurada y los registros de observación.

Momento (6): Sistematización de la información a la luz de los datos recolectados y articulación con la teoría.

Momento (7): Generación de resultados. Momento (8): Socialización de los hallazgos encontrados.

\section{Resultados}

A continuación, se presentan lo hallazgos del estudio teniendo en cuenta la pregunta problema: ¿Cuáles son los elementos que caracterizan la cultura afrocolombiana que se constituyen en una estrategia de educación en la IED San Juan de Palos Prieto?

Es importante sistematizar las experiencias educativas tal como lo afirma (Mejía, 1989). 


\begin{tabular}{|c|c|c|}
\hline $\begin{array}{l}\text { Categoría } \\
\text { de estudio }\end{array}$ & $\begin{array}{l}\text { Pregunta } \\
\text { orientadora }\end{array}$ & Discurso de los actores \\
\hline $\begin{array}{l}\text { Cultura } \\
\text { Afro }\end{array}$ & $\begin{array}{l}\text { ¿Qué } \\
\text { entiendes } \\
\text { por identidad } \\
\text { cultural? }\end{array}$ & $\begin{array}{l}\text { "Entiendo que la identidad cultural } \\
\text { son cosas que hacen que uno se } \\
\text { orgullezca porque es afrocolombiano y } \\
\text { no por el color sino por la honestidad" } \\
\text { "son las costumbres de un pueblo } \\
\text { sus culturas, son sus bailes, su } \\
\text { gastronomia, su dialecto" "entiendo } \\
\text { que es algo que nos identifica como } \\
\text { persona como las cualidades y } \\
\text { características" "los aspectos que uno } \\
\text { tienen entre un conjunto, pueblos, } \\
\text { culturas, costumbres que uno como } \\
\text { persona aprende". }\end{array}$ \\
\hline $\begin{array}{l}\text { Cultura } \\
\text { Afro }\end{array}$ & $\begin{array}{l}\text { ¿Cuáles son } \\
\text { los elementos } \\
\text { que hacen } \\
\text { parte de la } \\
\text { identidad } \\
\text { cultural del } \\
\text { pueblo afro? }\end{array}$ & $\begin{array}{l}\text { "las costumbres, los bailes, las comidas } \\
\text { y las costumbres que antes eran que en } \\
\text { semana santa hacian dulces y todas se } \\
\text { han perdido", "el dialecto", "el cabello, } \\
\text { el color de la piel", "las fiestas" "la } \\
\text { forma de vestir". }\end{array}$ \\
\hline
\end{tabular}

$\begin{array}{ll} & \text { ¿Dentro de } \\ & \text { la clase se } \\ \text { Estrategia } & \text { comparten o } \\ \text { educativa } & \text { viven rasgos } \\ & \text { propios de la } \\ & \text { cultura afro? } \\ & \text { ¿Cuáles? }\end{array}$

"Si. Porque dan catedra afro hablan que todos somos afro dependiendo su tipo de piel blanco, morenos, negros todos somos afro y muchos nos sentimos orgullosos" "Los profesores también son afro y más que saben cuáles son nuestros rasgos como afro" "Si. Porque algunos compañeros vienen de la cultura afrocolombiana y tienen costumbres afro".

"Que no permitieran que los otros estudiantes los maltraten, se burlen porque son negros" "Si me gustaría ¿Dentro de la clase se porque asi podemos saber porque aprendemos sobre la identidad comparten o cultural de los afrocolombianos pero

Estrategia Educativa viven rasgos propios de la cultura afro? ¿Cuáles? que la estudien de una manera que todos lo comprendemos, también por no a la discriminación" "Que fueron a un pueblo como en Palenque un pueblo que sus tradiciones siguen vivas que uno aprendiera un poco de nuestra cultura"

"Si. Porque todos somos territorio afro

$\begin{array}{ll} & \text { ¿Crees } \\ \text { Cultura } & \text { que los } \\ \text { Afro y } & \text { estudiantes } \\ \text { estrategia } & \text { valoran y } \\ \text { educativa } & \text { respetan la } \\ & \text { cultura afro? } \\ & \text { ¿Por qué? }\end{array}$
$y$ todos debemos conservar nuestras tradiciones" "Si. Porque como ellos también hacen parte de la raza afro para la cual se vuelven entre sí" "Si porque ellos saben que la identidad cultural es algo importante para los Afro y ellos respetan sus tradiciones y costumbres"
Articulación y sistematización teórica

De acuerdo a los hallazgos encontrados en la entrevista aplicada, se deduce que lo estudiantes en algún momento de su vida han vivido de alguna forma el concepto de identidad cultural.

Frente a esto para Molano (s.f.) "El concepto de identidad cultural encierra un sentido de pertenencia a un grupo social con el cual se comparten rasgos culturales, como costumbres, valores y creencias. La identidad no es un concepto fijo, sino que se recrea individual y colectivamente y se alimenta de forma continua de la influencia exterior".

Lo que realmente quiere decir Molano, es que la identidad cultural, guarda un sentido social en el que definan los rasgos culturales, sus dialectos, sus costumbres, sus valores y las creencias. Los estudiantes ven la identidad cultural como algo que los representa, que los muestra y que los identifica como comunidad, la cual deben de vivirla y sentirla para poder sentido de pertenencia.

Finalmente, a la luz de lo expresado por los estudiantes, éstos tienen claro que hace parte de la identidad cultural del pueblo afro y cómo ven la identidad cultural del pueblo afro, la cual rodea a la IED de San Juan de Palos Prieto. (Carvalho, 2002). Además, se ha acrecentado la visibilidad y apropiación de la cultura afro (tanto africana como diaspórica en América) en los centros urbanos occidentales. Han emergido diferentes prácticas de danza, música y religión, ofrecidas como experiencias para los jóvenes en los que en su mayoría hay una separación entre la circulación de los símbolos de africanidad y el destino contingente, histórico, de las comunidades afroamericanas.

La cultura de los pueblos se caracteriza por ser arraigada, y que una magna representación de las comunidades. Por lo tanto, perder las tradiciones es muy complejo, sin embargo la idea de la población es conservarla intacta con el pasar de los años.

Fuente: Elaboración propia. (2018). 


\section{Conclusiones}

Todos los hallazgos encontrados en el presente estudio permiten inferir que la comunidad académica en general reconoce la importancia conceptual y práctica de la cultura Afro, por lo que se logró una apropiación de los mismos por lo que se obtuvieron y transmitieron nuevos conocimientos en espacios creados para tal fin.

Se reconoce entre los estudiantes que los elementos de identidad cultural Afro, entre otros, son: la música, la gastronomía, la espiritualidad, la innovación espacial, vestimenta, mitología, tradiciones y en algunas regiones hasta el dialecto y el acento al hablar. Lo anterior lleva a promover el respeto por la diferencia cultural y la inclusión. Los estudiantes aprenden que preguntar para conocer es mejor que juzgar a través de distintas actividades aprenden a identificar y valorar los rasgos y aportes de la cultura Afro en Colombia

Finalmente, la contribución de valores tangibles y no tangibles de los pueblos africanos en la construcción de nuestra propia cultura es parte de nuestra realidad y por lo tanto muy notoria. La población Afro en Colombia representada aproximadamente el $26 \%$ del total nacional. La comunidad educativa de la IED San Juan de Palos Prieto resalta la importancia de celebrar el día de la Afro-Colombianidad dado que es una raza mestiza. Que el carácter Afro de la población mundial no es una cuestión exclusivamente de éstos, sino que es un patrimonio de la humanidad por lo que significa para el conocimiento universal.

\section{Referencias}

Andrade, M. (2011). El Afro en la Escuela: Cátedra de Estudios Afrocolombinos. Antioquia, Colombia. Obtenido de http://ayura.udea.edu.co:8080/ jspui/bitstream/123456789/219/1/ EB0271.pdf

Antón, J., Bello , Á., Del Popolo, F., Paixao, M., \& Rangel, M. (2005). Afrodescendientes en América Latina y el Caribe: Del reconocimiento estadístico a la realización de derechos. (CEPAL, Ed.) Chile. Obtenido de http://repositorio.cepal.org/bitstream/ handle/11362/7227/S0900315_ es.pdf?sequence $=1 \&$ isAllowed $=\mathrm{y}$.

Antón, J., Bello, Á., Del Popolo, F., Paixao, M., \& Rangel, M. (2009). Afrodescendientes en América Latina y el Caribe: Del reconocimiento estadístico a la realización de derechos ( S.09.II.G.47 ed.). (N. Unidas, Ed.) Santiago de Chile, Chile. Obtenido de http:// repositorio.cepal.org/bitstream/ handle/11362/7227/S0900315_ es.pdf?sequence $=1 \&$ isAllowed $=\mathrm{y}$

Avendaño, I., Cortés, O. y Guerrero, H. (2015) Competencias sociales y tecnologías de la información y la comunicación como factores asociados al desempeño en estudiantes de básica primaria con experiencia de desplazamiento forzado Diversitas: Perspectivas en Psicología, vol. 11, núm. 1, 2015, pp. 13-36 Universidad Santo Tomás Bogotá, Colombia.http://www.scielo.org.co/ pdf/dpp/v11n1/v11n1a02.pdf.

Bartomeu, F. (1997). Una sospechosa identidad. Perfiles Educativos, (75 ed., Vol. 19). (IRESIE, Ed.) 
Carvajal, Á. (2008). Teorías y modelos: Formas de representación de la realidad. Comunicación, 12(1). Obtenido de http://revistas.tec.ac.cr/index.php/ comunicacion/article/view/1212/1118

Carvalho, J. d. (2002). Las culturas Afroamericanas en Iberoamérica: lo negociable y lo innegociable. Brasilia, Brasil. Obtenido de http://www.nacionmulticultural.unam.mx/reconocimientopueblosnegros/docs/178.pdf

Chalá, J. (02 de Julio de 2006). La esclavización nos quitó hasta el nombre. Obtenido de https://www.eluniverso. com/2006/07/02/0001/12/61C7C9006 9B04D12A753A2C0D9A87FE2.html

García, J. (2001). Comunidades afroamericanas y transformaciones sociales. (C. L. Sociales, Ed.) Buenos Aires, Argentina: CLACSO. Obtenido de http://biblioteca.clacso.edu.ar/clacso/ gt/20100912035544/4garcia.pdf

Ianini, O. (1975). Esclavitud y capitalismo. Obtenido de http://marxsimoanticapitalista.blogspot.com.co/2013/04/ esclavitud-y-capitalismo-octavio-ianni.html

Mejía, J. (1989). Sistematizar nuestras prácticas educativas. (C. d. Popular, Ed.) Bogotá, Colombia. Obtenido de http://centroderecursos.alboan.org/ sistematizacion/es/registros/689-sistematizar-nuestras-practicas-educativas

Miguelez, M. M. (2006). La investigaciópn cualitativa (síntesis conceptual). Revista II PSI, 09, 123, 146. Obtenido de http://sisbib.unmsm.edu. pe/bvrevistas/investigacion_psicologia/v09_n1/pdf/a09v9n1.pdf

Mineducación. (2002). Cátedra de estudios Afrocolombianos. Bogotá, Colombia. Obtenido de https://www.mineducacion.gov.co/1759/articles-339975_recurso_2.pdf
Oliveira, D. d. (2001). Racismo Estructural - Apuntes para una Discusión Conceptual. (ALAI, Ed.) Irene León. Obtenido de https://repositorio.cepal. org/bitstream/handle/11362/7227/ S0900315_es.pdf;jsessionid=6D2C9 7AEB1EA490AE09F2EE466ACA7C $\mathrm{D}$ ?sequence $=1$

Palacios, E., Hurtado, O., \& Benítez, M. (2010). Aprender de la memoria cultural afrocolombiana. Valle del Cauca, Colombia. Obtenido de file:///C:/Users/ HP/Downloads/art\%C3\%ADculo_redalyc_99618003002.pdf

Rahier, J. (1998). Estudios de negros en la antropología ecuatoriana; presencia, invisibiidad y reproducción del orden racial espacial. (Vol. III). Quito, Ecuador.

Rangel, M. (2005). Una panorámica de las articulaciones y organizaciones de los afrodescendientes en América Latina y el Caribe. (N. Unidas, CEPAL, \& UNFPA, Edits.) Santiago de Chile, Chile. Obtenido de https:// repositorio.cepal.org/bitstream/ handle/11362/7227/S0900315_ es.pdf?sequence $=1 \&$ is Allowed $=y$

Sánchez, A. (2017). REPRESENTANDO "LO AFRO": CONSUMO CULTURAL DE DANZAS AFRICANAS POR PARTE DE PRACTICANTES BOGOTANOS. Universidad Distrital Francisco José de Caldas, 12(21). Obtenido de https://revistas.udistrital.edu.co/ojs/index.php/c14/article/ view/11900/html

Stavenhagen, R. (2001). El derecho de sobrevivencia: la lucha de los pueblos indígenas en América Latina contra el racismo y la discrimincaión. Santiago de chile, Chile. Obtenido de https://www.cepal.org/mujer/publicaciones/sinsigla/xml/6/6826/sobrevivencia_stavenhagen.PDF 
Torre, C. d. (1996). El racismo en Ecuador: Experiencias de los indios de clase media. (C. A. Popular, Ed.) Quito, Ecuador: CAAP. Obtenido de http:// biblioteca.clacso.edu.ar/Ecuador/ caap/20120928023907/torre.pdf
Wabgou, m. (2008). Migraciones internacionales y cambio social en las sociedades modernas. Bogotá, Colombia. Obtenido de https://revistas. unal.edu.co/index.php/recs/article/ view/9653/10219 